Vol. 44 (1991) [263-269]

\title{
ON THE GROWTH OF COMPOSITIONS OF LINEAR AND MEROMORPHIC FUNCTIONS
}

\author{
JIANYONG QIAO
}

Let $f(z)$ be a meromorphic function; we shall investigate the asymptotic behaviour of the ratio $T(r, f(z+\alpha)) / T(r, f(z))$ and $T(r, f(\alpha z)) / T(r, f(z))$, and discuss the growth of the meromorphic solutions of some functional equations.

\section{INTRODUCTION AND MAIN RESULTS}

We shall adopt the fundamental concepts and basic notation of Nevanlinna's theory in this paper. Let $f(z)$ be a meromorphic function and $T(r, f(z))$ be its Nevanlinna characteristic function. We denote the order and the lower order of $f(z)$ by $\rho_{f}$ and $\mu_{f}$ respectively in the sense of Nevanlinna. In addition, we put

$$
\widehat{\rho}_{f}=\limsup _{r \rightarrow \infty} \log \log T(r, f(z)) / \log r \text { and } \widehat{\mu}_{f}=\liminf _{r \rightarrow \infty} \log \log T(r, f(z)) / \log r
$$

$\hat{\rho}_{f}$ and $\hat{\mu}_{f}$ are said to be the hyperorder and lower hyperorder of $f(z)$ respectively.

It is obvious that $\hat{\rho}_{f}>0$ (or $\widehat{\mu}_{f}>0$ ) implies that $\rho_{f}=\infty$ (or $\mu_{f}=\infty$ ). Yang [1] proposed the following open problems:

Problem A: ([1], p.168). Let $f(z)$ be a meromorphic function and

$$
\lim _{r \rightarrow \infty} T(r, f(z+1)) / T(r, f(z))=\infty
$$

Can we conclude that $\mu_{f}=\infty$ ?

PROBLEM B: ([1], p.251). Let $f_{1}, f_{2}, g_{1}$ and $g_{2}$ be entire functions. Suppose that

$$
T\left(r, f_{1}\right) \sim T\left(r, f_{2}\right), \quad T\left(r, g_{1}\right) \sim T\left(r, g_{2}\right), \quad(r \rightarrow \infty) .
$$

Can we conclude that

$$
T\left(r, f_{1}\left(g_{1}\right)\right) \sim T\left(r, f_{2}\left(g_{2}\right)\right), \quad(r \rightarrow \infty) ?
$$

We shall give the answers to these two problems in this paper. Firstly, we have the following result

Received 19 October 1990

Copyright Clearance Centre, Inc. Serial-fee code: 0004-9729/91 \$A2.00+0.00. 
THEOREM 1. Let $f(z)$ be a meromorphic function such that $\widehat{\mu}_{f}<1, A_{j}(j=1,2, \ldots, m)$ and $B_{j}(j=1,2, \ldots, n)$ be positive real numbers, and $\alpha_{j}(j=1,2, \ldots, m)$ and $\beta_{j}(j=1,2, \ldots, n)$ be complex numbers. Then

$$
\liminf _{r \rightarrow \infty} \frac{\sum_{j=1}^{m} A_{j} T\left(r, f\left(z+\alpha_{j}\right)\right)}{\sum_{j=1}^{n} B_{j} T\left(r, f\left(z+\beta_{j}\right)\right)} \leqslant \frac{\sum_{j=1}^{m} A_{j}}{\sum_{j=1}^{n} B_{j}} \leqslant \limsup _{r \rightarrow \infty} \frac{\sum_{j=1}^{m} A_{j} T\left(r, f\left(z+\alpha_{j}\right)\right)}{\sum_{j=1}^{n} B_{j} T\left(r, f\left(z+\beta_{j}\right)\right)} .
$$

REMARK. (a) If (1) holds, then (2) does not hold for $m=n=1, A_{1}=B_{1}=1$, $\alpha_{1}=1$ and $\beta_{1}=0$. By Theorem 1 we have that $\hat{\mu}_{f} \geqslant 1$; thus $\mu_{f}=\infty$. This gives an affirmative answer to the Problem A.

(b) For $f(z)=e^{e^{z}}$, we can verify that $\widehat{\mu}_{f}=1$. It is easily seen that (2) does not hold for $m=n=1, A_{1}=B_{1}=1, \alpha_{1}=1$ and $\beta_{1}=0$. Therefore the conditions of Theorem 1 cannot be weakened.

Next we consider the asymptotic behaviour of the ratio $T(z, f(\alpha z)) / T(r, f(\beta z))$, and have

THEOREM 2. Let $f(z)$ be a meromorphic function, $\alpha$ and $\beta$ be two complex constants satisfying $|\alpha|>|\beta|>0$. Then

$$
\liminf _{r \rightarrow \infty} \frac{T(r, f(\alpha z))}{T(r, f(\beta z))} \leqslant\left|\frac{\alpha}{\beta}\right|^{\mu_{f}} \leqslant\left|\frac{\alpha}{\beta}\right|^{\rho_{f}} \leqslant \limsup _{r \rightarrow \infty} \frac{T(r, f(\alpha z))}{T(r, f(\beta z))} .
$$

REMARK. (a) Let $f_{1}, f_{2}$ be meromorphic functions, $\rho_{f_{1}}>0$ and

$$
T\left(r, f_{1}\right) \sim T\left(r, f_{2}\right), \quad(r \rightarrow \infty) .
$$

Put $g_{1}(z)=c z$ and $g_{2}(z)=z$; here $c$ is a complex constant and $|c|>1$. It is obvious that

$$
T\left(r, g_{1}\right) \sim T\left(r, g_{2}\right), \quad(r \rightarrow \infty) .
$$

But by Theorem 2 we have

$$
\underset{r \rightarrow \infty}{\limsup } \frac{T\left(r, f_{1}\left(g_{1}\right)\right)}{T\left(r, f_{2}\left(g_{2}\right)\right)}=\limsup _{r \rightarrow \infty}\left[\frac{T\left(r, f_{1}(c z)\right)}{T\left(r, f_{1}(z)\right)} \cdot \frac{T\left(r, f_{1}(z)\right)}{T\left(r, f_{2}(z)\right)}\right] \geqslant|c|^{\rho_{f_{1}}}>1 .
$$

Thus we give a negative answer to Problem $B$.

(b) Choose $f(z)=e^{z}$; then $\rho_{f}=\mu_{f}=1$. It is easily to verify that

$$
\lim _{r \rightarrow \infty} T(r, f(\alpha z)) / T(r, f(\beta z))=\left|\frac{\alpha}{\beta}\right| \text {. }
$$


Thus the result (3) of Theorem 2 is sharp.

Considering the functional equation

$$
\alpha_{n} f(z+n)+\alpha_{n-1} f(z+n-1)+\cdots+\alpha_{1} f(z+1)=R(f(z)),
$$

in which $R(w)=P(w) / Q(w), P(w)=a_{p} w^{p}+\cdots+a_{1} w+a_{0}$ and $Q(w)=b_{q} w^{q}+\cdots+$ $b_{1} w+b_{0}$ are supposed to be mutually prime, $\alpha_{n}, \ldots, \alpha_{1} ; a_{p}, \ldots, a_{1}, a_{0} ; b_{q}, \ldots, b_{1}, b_{0}$ are constants, and $\alpha_{n} a_{p} b_{q} \neq 0$, Yanagihara proved

Theorem A. [4] Suppose $\max (p, q) \geqslant n+1$. Then any non-constant meromorphic solution $f(z)$ of $(4)$ is of order $\rho_{f}=\infty$.

In this paper we generalise the above Theorem $A$ to the following Theorem 3 . Here we consider the functional equation

$$
\sum_{i=1}^{n} R_{i 1}\left(z, f\left(z+\alpha_{i 1}\right)\right) R_{i 2}\left(z, f\left(z+\alpha_{i 2}\right)\right) \ldots R_{i k}\left(z, f\left(z+\alpha_{i k}\right)\right)=R_{0}(z, f(z))
$$

where $\alpha_{i j}(i=1,2, \ldots, n ; j=1,2, \ldots, k)$ are constants, $R_{i j}(z, w)(i=1,2, \ldots, n$; $j=1,2, \ldots, k)$ and $R_{0}(z, w)$ are rational functions of the form $R(z, w)=$ $P(z, w) / Q(z, w) \quad P(z, w)=\sum_{j=1}^{p} a_{j}(z) w^{j}, Q(z, w)=\sum_{j=1}^{q} b_{j}(z) w^{j}$, in which $a_{j}(z)(j=0,1,2, \ldots, p)$ and $b_{j}(z)(j=0,1,2, \ldots, q)$ are polynomials. $P(z, w)$ and $Q(z, w)$ are supposed to be mutually prime. Denote $\partial R=\max (p, q)$. We have the following result.

Theorem 3. Suppose $\partial R_{0} \geqslant \sum_{i=1}^{n} \sum_{j=1}^{k} \partial R_{i j}+1$. Then any transcendental meromorphic solution $f(z)$ of $(5)$ satisfies $\hat{\mu}_{f} \geqslant 1$.

Considering the equation of Schröder,

$$
f(c z)=Q(f(z))
$$

in which $Q(z)$ is a polynomial of degree $n$ and $c$ is a constant satisfying $|c|>1$, Shimomura proved

TheOREM B. [3] Suppose $f(z)$ is a non-constant entire solution of (6); then the order $\rho_{f}=\log n / \log |c|$.

Theorem C. [3] If $|c|<1$, then (6) has no non-constant entire solution.

In this paper we generalise the above theorems to the following Theorem 4. Here we consider the functional equation

$$
R_{1}(z, f(c z))=R_{2}(z, f(z)),
$$

in which $R_{j}(z, w)(j=1,2)$ have the same form as the above $R(z, w)$, and $c$ is a constant satisfying $|c|>1$. We have the following result: 
THEOREM 4. (a) If $\partial R_{2} \geqslant \partial R_{1}$, and $f(z)$ is a transcendental meromorphic solution of (7), then

$$
\rho_{f}=\mu_{f}=\log \frac{\partial R_{2}}{\partial R_{1}} / \log |c| .
$$

(b) If $\partial R_{2}<\partial H_{1}$, then (7) has no transcendental meromorphic solution.

\section{Proof of Theorem 1}

Firstly, without loss of generality, we suppose $\left|\alpha_{1}\right|=\max \left(\left|\alpha_{1}\right|,\left|\alpha_{2}\right|, \ldots\left|\alpha_{m}\right|\right)$, $\left|\beta_{1}\right|=\max \left(\left|\beta_{1}\right|,\left|\beta_{q}\right|, \ldots,\left|\beta_{n}\right|\right)$ and put $t=\left|\alpha_{1}\right|+\left|\beta_{1}\right|$. If $t=0$, then (2) obviously holds.

Below, we suppose $t>0$ and put

$$
\Omega=\liminf _{r \rightarrow \infty} \sum_{j=1}^{m} A_{j} T\left(r, f\left(z+\alpha_{j}\right)\right) / \sum_{j=1}^{n} B_{j} T\left(r, f\left(z+\beta_{j}\right)\right),
$$

( $\Omega$ is finite or infinite). If $\Omega=0$, then $\Omega<\sum_{j=1}^{m} A_{j} / \sum_{j=1}^{n} B_{j}$ holds. Next, we suppose $\Omega>0$. Thus for any positive number $\sigma<\Omega$, there exists $r_{1}>0$ such that

$$
\sum_{j=1}^{m} A_{j} T\left(r, f\left(z+\alpha_{j}\right)\right)>\sigma \sum_{j=1}^{n} B_{j} T\left(r, f\left(z+\beta_{j}\right)\right),
$$

when $r \geqslant r_{1}$. We choose a number $a$ which is not a Valiron deficient value of $f\left(z+\alpha_{j}\right)(j=1,2, \ldots, m), f\left(z+\beta_{j}\right)(j=1,2, \ldots, n)$ and $f(z)$. Therefore for any $\varepsilon>0$, there exists $r_{2}>0$ such that the following four inequalities hold when $r \geqslant r_{2}$.

$$
\begin{aligned}
& T\left(r, f\left(z+\alpha_{j}\right)\right) \leqslant(1+\varepsilon) N\left(r, f\left(z+\alpha_{j}\right)=a\right),(j=1,2, \ldots, m) \\
& T\left(r, f\left(z+\beta_{j}\right)\right) \geqslant(1-\varepsilon) N\left(r, f\left(z+\beta_{j}\right)=a\right),(j=1,2, \ldots, n) \\
& N\left(r+\left|\alpha_{1}\right|, f(z)=a\right) \leqslant(1+\varepsilon) T\left(r+\left|\alpha_{1}\right|, f(z)\right) \\
& N\left(r-\left|\beta_{1}\right|, f(z)=a\right) \geqslant(1-\varepsilon) T\left(r-\left|\beta_{1}\right|, f(z)\right)
\end{aligned}
$$

It is obvious that

$$
N\left(r, f\left(z+\alpha_{j}\right)=a\right) \leqslant N\left(r+\left|\alpha_{j}\right|, f(z)=a\right) \leqslant N\left(r+\left|\alpha_{1}\right|, f(z)=a\right) .
$$

Hence it follows from (9), (11) and the above inequality that

$$
T\left(r, f\left(z+\alpha_{j}\right)\right) \leqslant(1+\varepsilon)^{2} T\left(r+\left|\alpha_{1}\right|, f(z)\right), \quad(j=1,2, \ldots, m)
$$


when $r \geqslant r_{2}$. We can also have

$$
N\left(r, f\left(z+\beta_{j}\right)=a\right) \geqslant N\left(r-\left|\beta_{j}\right|, f(z)=a\right) \geqslant N\left(r-\left|\beta_{1}\right|, f(z)=a\right) .
$$

Hence it follows from (10), (12) and the above inequality that

$$
T\left(r, f\left(z+\beta_{j}\right)\right) \geqslant(1-\varepsilon)^{2} T\left(r-\left|\beta_{1}\right|, f(z)\right), \quad(j=1,2, \ldots, n)
$$

when $r \geqslant r_{2}$. So (8), (13) and (14) yield that

$$
T\left(r+\left|\alpha_{1}\right|, f(z)\right)>A T\left(r-\left|\beta_{1}\right|, f(z)\right)
$$

when $r \geqslant \max \left(r_{1}, r_{2}\right) ;$ here $A=\sigma((1-\varepsilon) /(1+\varepsilon))^{2} \sum_{j=1}^{n} B_{j} / \sum_{j=1}^{m} A_{j}$. Put $r_{0}=$ $\max \left(r_{1}, r_{2}\right)+\left|\beta_{1}\right|$. It follows that

$$
T(r+t, f(z))>A T(r, f(z)),
$$

when $r \geqslant r_{0}$.

Suppose $\Omega>\sum_{j=1}^{m} A_{j} / \sum_{j=1}^{m} B_{j}$ Then we can choose suitable $\sigma$ and $\varepsilon$ such that $A>1$. From (15) we easily deduce

$$
T\left(r_{0}+k t, f(z)\right)>A^{k} T\left(r_{0}, f(z)\right),
$$

in which $k$ is any natural number. For an arbitrarily real number $r \geqslant r_{0}$, we assume $r \in\left[r_{0}+k t, r_{0}+(k+1) t\right)$ and obtain that

$$
T(r, f(z)) \geqslant T\left(r_{0}+k t, f(z)\right)>A^{k} T\left(r_{0}, f(z)\right)>A^{\left(r-r_{0}-t\right) / t} T\left(r_{0}, f(z)\right) .
$$

It follows that $\hat{\mu}_{f} \geqslant 1$. This is a contradiction. Therefore $\Omega \leqslant \sum_{j=1}^{m} A_{j} / \sum_{j=1}^{n} B_{j}$. The following inequality is thus proved.

$$
\underset{r \rightarrow \infty}{\liminf } \sum_{j=1}^{m} A_{j} T\left(r, f\left(z+\alpha_{j}\right)\right) / \sum_{j=1}^{n} B_{j} T\left(r, f\left(z+\beta_{j}\right)\right) \leqslant \sum_{j=1}^{m} A_{j} / \sum_{j=1}^{n} B_{j} .
$$

By the same method we can also prove

$$
\liminf _{r \rightarrow \infty} \sum_{j=1}^{n} B_{j} T\left(r, f\left(z+\beta_{j}\right)\right) / \sum_{j=1}^{m} A_{j} T\left(r, f\left(z+\alpha_{j}\right)\right) \leqslant \sum_{j=1}^{n} B_{j} / \sum_{j=1}^{m} A_{j} .
$$

This implies that

$$
\underset{r \rightarrow \infty}{\limsup } \sum_{j=1}^{m} A_{j} T\left(r, f\left(z+\alpha_{j}\right)\right) / \sum_{j=1}^{n} B_{j} T\left(r, f\left(z+\beta_{j}\right)\right) \geqslant \sum_{j=1}^{m} A_{j} / \sum_{j=1}^{n} B_{j} .
$$

which, together with (16), proves (2). The proof of Theorem 1 is complete 


\section{Proof of Theorem 2}

At first we put

$$
\Omega=\liminf _{r \rightarrow \infty} T(r, f(\alpha z)) / T(r, f(\beta z)) ;
$$

( $\Omega$ is finite or infinite). If $\Omega \leqslant 1$, it is obvious that $\Omega<\left|\frac{\alpha}{\beta}\right|^{u_{f}}$. Below, we suppose $\Omega>1$. Then for any positive number $\sigma<\Omega$, there exists $r_{1}>0$ such that

$$
T(r, f(\alpha z))>\sigma T(r, f(\beta z))
$$

when $r \geqslant r_{1}$. We choose a number $a$ which is not a Valiron deficient value of $f(\alpha z)$ and $f(\beta z)$. Thus for any $\varepsilon>0$, there exists $r_{2}>0$ such that the following inequality holds when $r \geqslant r_{2}$.

$$
\begin{aligned}
T(t, f(\alpha z)) & \leqslant(1+\varepsilon) N(r, f(\alpha z)=a)=(1+\varepsilon) N\left(\left|\frac{\alpha}{\beta}\right| r, f(\beta z)=a\right) \\
& \leqslant(1+\varepsilon)^{2} T\left(\left|\frac{\alpha}{\beta}\right| r, f(\beta z)\right) .
\end{aligned}
$$

Put $t=|\alpha / \beta|>1$ and $r_{0}=\max \left(r_{1}, r_{2}\right)$. It follows from (17) and (18) that

$$
T(t r, f(\beta z))>A T(r, f(\beta z)),
$$

when $r \geqslant r_{0}$; here $A=\sigma /(1+\varepsilon)^{2}$. Since $\Omega>1$, we can choose suitable $\sigma$ and $\varepsilon$ such that $A>1$. Hence (19) implies

$$
T\left(r_{0} t^{k}, f(\beta z)\right)>A^{k} T\left(r_{0}, f(\beta z)\right),
$$

in which $k$ is any natural number. For an arbitrarily real number $r \geqslant r_{0}$, we assume $r \in\left[t^{k} r_{0}, t^{k+1} r_{0}\right)$. By the same method as in the proof of Theorem 1 we can deduce $\mu_{f} \geqslant \log A / \log t$. Making $\varepsilon \rightarrow 0$ and $\sigma \rightarrow \Omega$, we obtain $\mu_{f} \geqslant \log \Omega / \log t$, that is,

$$
\liminf _{r \rightarrow \infty} T(r, f(\alpha z)) / T(r, f(\beta z)) \leqslant\left|\frac{\alpha}{\beta}\right|^{\mu_{f}} .
$$

By a similar method we can also prove that

$$
\underset{r \rightarrow \infty}{\limsup } T(r, f(\alpha z)) / T(r, f(\beta z)) \geqslant\left|\frac{\alpha}{\beta}\right|^{\rho_{f}} .
$$

Theorem 2 is thus proved. 


\section{Proof of Theorem 3 and Theorem 4}

In order to prove Theorem 3 and Theorem 4, we need the following

LemMa. [2] Suppose $R(z, w)$ is defined as before, and $f(z)$ is a meromorphic function. Then

$$
T(r, R(z, f(z)))=\partial R \cdot T(r, f(z))+O(\log r) .
$$

Proof of Theorem 3: Firstly, the following inequality follows from (5) and the above lemma.

$$
\partial R_{0} T(r, f(z)) \leqslant \sum_{i=1}^{n} \sum_{j=1}^{k} \partial R_{i j} T\left(r, f\left(z+\alpha_{i j}\right)\right)+O(\log r) .
$$

If $\widehat{\mu}_{f}<1$, since $f(z)$ is transcendental, then we deduce from (20) and Theorem 1 that $\partial R_{0} \leqslant \sum_{i=1}^{n} \sum_{j=1}^{k} \partial R_{i j}$. This is a contradiction. Theorem 3 is thus proved.

Proof of Theorem 4: (a) By the above lemma and (7) we have

$$
\partial R_{1} T(r, f(c z))=\partial R_{2} T(r, f(z))+O(\log r) .
$$

It follows from Theorem 2 and (21) that $|c|^{\rho_{f}}=|c|^{\mu_{f}}=\partial R_{2} / \partial R_{1}$, that is, $\rho_{f}=\mu_{f}=$ $\log \left(\partial R_{2} / \partial R_{1}\right) / \log |c|$.

(b) Suppose (7) has a transcendental meromorphic solution $f(z)$; then by the above lemma and (7) we can deduce (21). By Theorem 2 we have $\partial R_{2} \geqslant|c|^{\rho_{f}} \partial R_{1} \geqslant \partial R_{1}$. This is a contradiction. Theorem 4 is thus proved.

\section{REFERENCES}

[1] C.T. Chang and C.C. Yang, "Theory of fix-points and factorization of memomorphic functions', (in Chinese), in Mathematical Monograph Series (Peking University Publishing House, 1988).

[2] F. Gackstatter and I. Laine, 'Zur theorie der gewöhnlichen differeentialgleichungen in komplexen', Ann. Polon. Math. 38 (1980), 259-287.

[3] S. Shimomura, 'Entire solutions of a polynomial difference equation', J. Fac. Sci. Univ. Tokyo Sect. IA Math. 28 (1981), 253-266.

[4] N. Yanagihara, 'Meromorphic solutions of some difference equations of higher order, I', Proc. Japan Acad. 58A (1982), 21-24. 'II', 284-286 .

\footnotetext{
Department of Mathematics

Huaibei Meitan Teachers College

Huaibei, Anhui Province

Peoples Republic of China
} 